\title{
ERGONOMIC EVALUATION AND RISK MANAGEMENT OF MANUAL MATERIAL HANDLING ACTIVITIES
}

\author{
Amit Waman Bankar ${ }^{1}$, Dr. Bhushan Ratnakar Mahajan ${ }^{2}$ \\ ${ }^{1,2} \mathrm{JD}$ College of engineering and management, Nagpur, India \\ ${ }^{1}$ amitb9275@gmail.com, ${ }^{2}$ brmahajan2003@yahoo.co.in
}

\begin{abstract}
Manual material handling follows in nearly all working surroundings, although labors in agriculture fields, construction sites, restaurants, and hotels area are likely to be working with heavy jobs in terms of weight, currencies, Manual handling of the heavy load may turn in to a growing disorder due to the steady or increasing drop of the musculoskeletal system due to constant material lifting / handling events. It may also reason to acute trauma like bone fractures or skin cuts from accidents. One must begin to identify the correct process of methods to deal with each material to solve the problem. Adherence to standard procedures for long-term resolution of the long-term effects caused by incorrect manual material handling, is addressed through specially designed workouts after or before work and, ultimately, also automated technology Or involved in providing assistance through manual tools that assist the user in dealing with content. The proposed task is to conduct studies to identify process issues and future results and to provide solutions to the industry by means of analysis and following standard procedures. This paper mainly deals with issues directly or indirectly in many aspects and discusses solutions implemented to deal with manual content in various industry. The study concluded with a novel automation solution for rapid analysis of effects due to manual analysis that could take years to identify.
\end{abstract}

Keywords: Manual Material Handling (MMH), Musculoskeletal Disorders (MSDs), Exoskeleton, Ergonomics.

\section{Introduction}

Manual Material Handling (MMH) is work events, which has a direct effect on the body/physical phase of employees in the manufacturing or production industry, it is essential to examine the risk [1]. In a rapid developing country like India, where different industrial operations are labor-intensive, semiautomated and which may include manual material handling $(\mathrm{MMH})$ in different conditions; Ergonomic evaluation is most essential to identify important heavy work load tasks. It is also necessary to evaluate customary working methods and attain more efficient and feasible methods of working[24]. Jobs that needs long-term manual work such as construction site jobs, service industry jobs, production unit jobs, physical assessment of the cardiovascular capacity can be used to determine a worker's capacity of physical fitness for work. To deal with the stated issues, various approaches, such as physiological, biomechanical, and physical assessment, can be used to appropriately reduce and reduce the risks of Musculoskeletal-Disorder (MSD) and to reduce a variety of other occupational injuries involving the material can be reduced. Ergonomic assessment / measurement tools deliver objective data about the physical or actual demands of manually handling tasks which will assist the user to develop an actual prevention measures and strategy.

Manual work handling rule and regulations establish a outline for establishments to avoid or reduce manual work or material handling activity. Employers must needs to evaluate work environment manual work handling processes and take steps to avoid or minimize the risk of physical injuries. Working environment threat can be avoided or minimized by introducing proper administrative procedures, such as a work area improvements to reduce avoidable long distances works or the use of suitable means in specialized mechanical apparatus [25]. Working risk physical assessment is a process that involves obtaining a detailed analysis of a task, gathering all the relevant technical and logical information of the task, and identifying if risks / hazards are existing, which ones to mitigate or eliminate the risk. Establish a plan to implement the factors/hazards and the granted governor processes [2]. As already mentioned, the risk physical assessment method should preferably be carried out in the design phase of a project implementation so that all possible efforts and redesigning can be made at that level to take the process below includes hazard identification, implementation of control measures, and risk assessment. This will supports the approach to effectively calculate manual working handling activity; It is essential to study the handling work in detail, collect relevant data, identify and assess risk factors / hazards in relation to the plan in directive, and then implement suitable answers or governor measures. 


\section{Research study objective}

Multiple sub fields and the discussion are available in the form of research papers or the industry standards manuals about, Manual Material Handling (MMH) and the issues occurred due to MMH. In order to understand the MMH the research or study flow should be in multiple dimensions and it should clear the background of the issues and reason for the same. The objective of the research study are mainly as following;

- Understanding of the ergonomics and its risk factors in $\mathrm{MMH}$

- $\quad$ Study of the existing methods and tools available for MMH assessment

- $\quad$ Listing the Musculoskeletal Disorder assessment tools and its comparison

- Understanding the utilization of the exoskeleton to assist workers in case of MMH

- $\quad$ Proposing the possible solution for performing the $\mathrm{MMH}$ assessment using manual and automation tools

The main reason for selecting these subchapter for study because, in order to start the study or the development in MMH researcher must be clear related to sub fields and the available literatures or the tools in this area. This paper gradually take the reader towards the guidance to selecting next path of improvisation in the field

\section{Research Methodology}

The information shared in the paper are collected from different sources like research papers, industrial standards manuals, white papers and the discussion session with the industry expert in local region. Following are the methodology utilized for the data collection and re-presentation in the paper.

- Data gathering: Collection of the data from multiple sources including the literature and the on-field information.

- Data unification: Structuring the data and removal of repeating information available from multiple sources.

- Data optimization: Re-structuring the information with checking its feasibility and the relevance with the selected title of the paper.

- Data sequencing: Compiling the information in the form of waterfall model which will gradually improvise and understanding of the reader in the $\mathrm{MMH}$ field

\section{Ergonomic Risk Factors}

Ergonomic in working risk factors are typically found in $\mathrm{MMH}$ environments. Ergonomic risk factors are the glitches in the manual working environment that cause preventable physical fatigue [10]. An active ergonomics enhancement procedure confirms that work and tasks are within the physical capabilities of the worker [3]. It is about detecting these ergonomic risk factors in industrial work atmosphere and putting governor measures in place to limit contact and this process would be the foundation of manual material handling (MMH) operations to prevent injuries [8]. The ergonomically designed work atmosphere reduces worker fatigue, weakness and discomfort.

2.1 Awkward postures: Employees ignores the proper posturing and working in unusual awkward postures like bending, twisting these may lead in breaks in internal tissues over the period of time [4].

2.2 Highly repetitive motions: As per the industry work allotment, employees employee perform similar task for multiple time or repetitive motions. This will benefited in building muscle memory and improve work efficiency but its adverse effect may lead in long term weakness in other part of the body and inability in different work execution [5].

2.3 Forceful exertions: Forcibly or unknowingly employee perform activity which may be out of capacity of the individual body. This is the most dangers risk factory in manual material handling, where it could quick damage to the body and with wrong posture execution it may damage body for long term [2].

2.4 Static posture: Static posture impact on body is very common among every industries. It is not specifically limited to manual material handling but applicable to almost every seating job works. This risk factor may affect body over the period of time. Simple break form work or stretching exercises between the works are the solutions to these type of risks [1].

2.5 Pressure points: This type of risk happen due to grasping loads or pressured contact with loads, leaning against materials, hard surfaces with sharp edges. This practice may damage tissues in quick time only. Repetitive practice may lead to permanent damages in body or injuries at particular points

\section{MMH Assessment}

The very first step in reducing the vulnerability related to Manual Material Handling (MMH) tasks is to examine the jobs to recognize the ergonomics hazards existing in a work environment [11]. The examination consisted of a different types of activities which may include observing the working environment worker, interviewing the workers and conferring the work with them, measuring the space involved Tasks, calculating the frequency of carrying tasks, etc. Through the analysis user can better identify the need of the procedural changes to follow in industry due to impact of manual material handling [12]. These analysis tools observe the different posture or the muscular joints of the body and take result from actual employee.

3.1 Nordic Body Map (NBM): This is individual identification survey that is used to recognize discomfort or complaints from individual's body parts while execution work environment activities. NBM is built on keeping 28 points of the body in consideration to evaluate it, based on the depth of complaints, are distributed into 4 levels of objections [6]. 
3.2 Rapid Entire Body Assessment (REBA): This method was primarily developed to examine the risks and issues experienced by workers depending on body posture while performing the work activities. These analysis note the different working postures of employees and get the inputs from the employee about its comfort or the discomfort of the posture over the period of time or quick impact [7].

3.3 Maximum Permissible Limit (MPL): This procedure in the fields of biomechanics that can be used as a splendid contraption for expressly lifting work activity is named as Maximum reasonable cutoff (MPL). This is dominatingly used to portray the degree of the applied Force Compression $(\mathrm{Fc})$ at the L5/S1. MPL is a limitation of the volume of compressive force on the piece L5/S1 and these are standardized by NIOSH (National Institute of Occupational Safety and Health) in the hour of 1981. As per the NIOSH appraisal, most indispensable level of compressive force on L5/S1 is $6400 \mathrm{~N}$, while range for standard force (Action Limit) is $3400 \mathrm{~N}$ on the piece L5/S1 [6]. 3.4 Stopwatch (Time Study): This structure is the lively assessment of working time routinely sought after the positions/work of brief period and which would be somber. Time study has obvious tremendous viewpoint like position, weight, weight or the relationship with follow for unequivocal time span [6].

3.5 Recommended Weight Limit (RWL): It portrays as the best store of the commitment that taking everything into account, any strong workers can lift all through a fundamental time period likely eight hours dependably, without an elevated peril of creating lower back torture. As demonstrated by the evaluation the best significant load to be lifted with both the hands and under the ideal conditions, is around 23.1 $\mathrm{Kg}$ [8].

Taking a blueprint of material overseeing affiliations, where most indispensable activities are act in a standing position. Workers reliably stay with flexed neck for 8-9 hrs as a piece of little by little work. While working hours workers get least breaks depending upon the consigned responsibility yet this breaks time are not worthy to offer rest to workers bodies. Table 1 portrays the body part influenced transparency related with persevering standing position work. Thusly, there was a shocking need to change the workstation so the responsiveness at neck, wrist/hand, shoulder and back could be limit.

\begin{tabular}{|c|c|c|c|c|}
\hline \multirow{2}{*}{ Body parts } & \multicolumn{4}{|c|}{ Exposure level (scores) } \\
\hline & Low & Moderate & High & Very high \\
\hline Back (static) & $8-15$ & $16-22$ & $23-29$ & $29-40$ \\
\hline Back (moving) & $10-20$ & $21-30$ & $31-40$ & $41-56$ \\
\hline Shoulder/arm & $10-20$ & $21-30$ & $31-40$ & $41-56$ \\
\hline Wristhand & $10-20$ & $21-30$ & $31-40$ & $41-46$ \\
\hline Neck & $4-6$ & $8-10$ & $12-14$ & $16-18$ \\
\hline
\end{tabular}

Table 1: Categorization of body part exposure

\section{Musculoskeletal Disorder}

When all is said in done, all representatives who working with static and little developments for a significant stretch of time in their every day working exercises would be at the danger for wounds including muscle issues or Musculoskeletal Disorders (MSDs)[9]. These are the deformities set off by development of wounds or the harm to the musculoskeletal framework, because of monotonous injury, which may shaping extensive harm and cause the torment. MSDs are a bunch of manifestations related to ligaments, tendons, muscle tissue, sensory system, ligaments, veins or the bone construction. MSDs essentially may cause shivering, torment, shaking, deadness, expanding, solidness, rest aggravation, or the consuming. Musculoskeletal grievances are the complaint with respect to the human body skeletal muscle felt by a distinctive individual beginning from gentle issues to major difficult issues.

Table 2 describes the features of different ergonomics analysis indices for the manual material handling activities performed at different industries.

\begin{tabular}{llllllll}
\hline Feature & NIOSH & $\begin{array}{l}\text { Snook } \\
\text { \& } \\
\text { Ciriello }\end{array}$ & OCRA & $\begin{array}{l}\text { Strain } \\
\text { Index }\end{array}$ & OWAS & RULA & REBA \\
& & & $\mathrm{x}$ & $\mathrm{x}$ & $\mathrm{x}$ & $\mathrm{x}$ & $\mathrm{x}$ \\
\hline Posture & & & $\mathrm{x}$ & $\mathrm{x}$ & & $\mathrm{x}$ & $\mathrm{x}$ \\
Upper limbs & & & & & $\mathrm{x}$ & $\mathrm{x}$ & $\mathrm{x}$ \\
Lower limbs & & & $\mathrm{x}$ & $\mathrm{x}$ & & $\mathrm{x}$ & $\mathrm{x}$ \\
Spine & $\mathrm{x}$ & $\mathrm{x}$ & $\mathrm{x}$ & $\mathrm{x}$ & $\mathrm{x}$ \\
Quantitative & $\mathrm{x}$ & $\mathrm{x}$ & & $\mathrm{x}$ & & $\mathrm{x}$ & $\mathrm{x}$ \\
Load/Force & $\mathrm{x}$ & $\mathrm{x}$ & $\mathrm{x}$ & $\mathrm{x}$ & & & \\
Frequency & $\mathrm{x}$ & $\mathrm{x}$ & $\mathrm{x}$ & $\mathrm{x}$ & & & \\
Duration & & $\mathrm{x}$ & $\mathrm{x}$ & & & & \\
Recovery & & & & & & & \\
\hline
\end{tabular}

Table 2: Features of the ergonomic assessment techniques used in MMH.

4.1 Cornell Musculoskeletal Discomfort Questionnaires (CMDQ): These Questionnaires have been initially developed under the supervision of Dr. Alan Hedge with other ergonomics field graduate students studying at Cornell University. These questionnaires are mainly centered on earlier presented research studies of musculoskeletal dis-order among industrial workers. Scoring of the questionnaires must be self-identified to anyone aware with these type of research. These mainly deal in finding out the symptoms of MSDs in individual employee and group of employee [9].

4.2 Rapid Upper Limb Assessment (RULA): This RULA was primarily developed as name suggested "Rapidly" assess the exposure of every volunteer workers to ergonomic risk factors mainly associated with physical upper extremity MSD. The RULA ergonomics valuation tool studies biomechanical or the physical postural load necessities of job tasks/demands on the neck, trunk, or the upper body [7].

4.3 Rapid Entire Body Assessment (REBA): REBA was again primarily developed as name suggested "Rapidly" evaluate 
risk of Musculoskeletal-Disorders (MSD) related with certain job tasks. The Mentioned REBA tool uses a methodical process to assess both upper and lower parts of the musculoskeletal system in biomechanical and MSD risks associated with the job task being assessed. A single page input worksheet could be used to assess essential or selected posture, type of movement or action, body forceful exertions, coupling, and repetition [7].

4.4 Ovako Working Posture Analyzing System (OWAS): This is a primarily basic observation technique / method for body postural analysis, but as per the study there has been no actual use in the building construction industry. This system was used to assess the working load in the repairing process of melting furnaces industry

\section{Exoskeleton}

Automation and robotics have made material handling easy however, there are major boundaries and few tasks cannot be done with the help of robots or are simply cost excessive. There are devices and technology like IADs or the exoskeletons are available and fully functional to assist workers in manual material handling [13], [14]. Developing and deploying exoskeleton are easy and helps workers in different activities like lifting high weight, relaxing muscles, climb stairs multiple time, standing for long time, or moving object from place to place. [15], [16], and [17] Even though exoskeletons are better technological solution in manual material handling but it also has some challenges like:

Availability: Very few companies have claimed for fully functional production exoskeletons for industries that result in to low availability [18].

Price: With the market ratio of demand vs supply the price of the exoskeletons are higher at this time which mar reduce depending upon the actual production of the suits [19].

Battery: Almost every exoskeleton designing companies or the research center are facing problem with power source to exoskeleton. Since the exoskeleton works on either hydraulic or the high torque motors and which both need high power source [20].

\section{Summary}

Proposed Study work deal with the understanding and study the impact of wrong practice of manual material handling in industry and providing the solution in the form of new methods for material handling and the preventions to be taken while performing the material handling process in working hours. The study will help industry, employee and the employer in multiple ways and the study will be able to help reducing the use of medical services due lowering the ratio of effect of body due to wrong MMH. The implementation of the study will help to ensuring compliance with legislation. Following mentioned are some of the benefits expected from proposed work outcome.
The production unit of medium scale industry in India will be able to understand the cause and effect of occupational hazard due to manual material handling and the employer will be able to provide info about physical demands of manual material handling tasks that may help the user to improve an effective prevention strategy. Employers will be able to carry out actual risk assessments, and finally take action to protect workers from the risks of issues occurred due to manual handling. The execution of MMH guidelines would help in decreasing absenteeism, employee turnover, or the retraining and low efficiency levels causing from working with discomfort. The study will help in decreasing or preventing injuries thereby lowering costs by eliminating Workers Compensation Board (WCB) claims and the worker will be able understand its safe and permissible capacity in MMH. The study will be able to help in employee morale, increasing productivity, as well as service and product quality.

\section{Conclusion}

Since the ergonomics evolution may help in identifying the different issues in the working environment and even different analysis tools may help in finding out the improvisation points, but still it needs the solution to reduce the problems in near future. There must be proper implementation at the level of technology, preventive measures or the time based exercise. This study was carried out to find out the possible solutions for preventive measure and to give solutions to avoid long term side effect due to manual material handling in different environment. Further study will be carried out to identify same assessment in large unorganized small industries all over the country.

\section{References}

[1] Thomas Erl, Ricardo Puttini, Zaigham Mahmood "Cloud Computing: Concepts, Technology \& Architecture" Prentice Hall, 2013

[2] Gururaj Ramachandra, Mohsin Iftikhar, Farrukh Aslam Khan, A Comprehensive Survey on Security in Cloud Computing, Procedia Computer Science, Volume 110, Pages 465-472, 2017.

[3] Shah, Krutika \& Vadiya, Vahida \& Jhaveri, Rutvij. A Survey Paper on Security in Cloud Computing: A Bibliographic Analysis. Circulation in Computer Science. vol. 1, pages-19-23, 2016.

[4] D. Zhe, W. Qinghong, S. Naizheng and Z. Yuhan, "Study on Data Security Policy Based on Cloud Storage," 2017 ieee 3rd international conference on big data security on cloud (bigdatasecurity), ieee international conference on high performance and smart computing (hpsc), and ieee international 
conference on intelligent data and security (ids), Beijing, 2017, pp. 145-149.

[5] A. Sun, G. Gao, T. Ji and X. Tu, "One Quantifiable Security Evaluation Model for Cloud Computing Platform," 2018 Sixth International Conference on Advanced Cloud and Big Data (CBD), Lanzhou, 2018, pp. 197-201.

[6] J. Shen, T. Zhou, D. He, Y. Zhang, X. Sun and Y. Xiang, "Block Design-Based Key Agreement for Group Data Sharing in Cloud Computing," in IEEE Transactions on Dependable and Secure Computing, vol. 16, no. 6, pp. 996-1010, 1 Nov.Dec. 2019.

[7] V. P. Lalitha, M. Y. Sagar, S. Sharanappa, S. Hanji and R. Swarup, "Data security in cloud," 2017 International Conference on Energy, Communication, Data Analytics and Soft Computing (ICECDS), Chennai, 2017, pp. 36043608.

[8] L. Qing, Z. Boyu, W. Jinhua and L. Qinqian, "Research on key technology of network security situation awareness of private cloud in enterprises," 2018 IEEE 3rd International Conference on Cloud Computing and Big Data Analysis (ICCCBDA), Chengdu, 2018, pp. 462-466.

[9] M. Nanda, A. Tyagi, K. Saxena and N. Chauhan, "Hindrances in the security of Cloud Computing," 2016 6th International Conference - Cloud System and Big Data Engineering (Confluence), Noida, 2016, pp. 193-198.

[10] Y. Reddy, "Big Data Security in Cloud Environment," 2018 IEEE 4th International Conference on Big Data Security on Cloud (BigDataSecurity), IEEE International Conference on High Performance and Smart Computing, (HPSC) and IEEE International Conference on Intelligent Data and Security (IDS), Omaha, NE, 2018, pp. 100-106.

[11] R. A. R. Shaikh and M. M. Modak, "Measuring Data Security for a Cloud Computing Service," 2017 International Conference on Computing, Communication, Control and Automation (ICCUBEA), Pune, 2017, pp. 1-5.

[12] P. Sha and Z. Zhu, "The modification of RSA algorithm to adapt fully homomorphic encryption algorithm in cloud computing," 2016 4th International Conference on Cloud Computing and Intelligence Systems (CCIS), Beijing, 2016 pp. 388-392.

[13] X. Song and Y. Wang, "Homomorphic cloud computing scheme based on hybrid homomorphic encryption," 2017 3rd IEEE International Conference on Computer and Communications (ICCC), Chengdu, 2017, pp. 2450-2453.

[14] Salma, R. F. Olanrewaju, K. Abdullah, Rusmala and H. Darwis, "Enhancing Cloud Data Security Using Hybrid of Advanced Encryption Standard and Blowfish Encryption Algorithms," 2018 2nd East Indonesia Conference on Computer and Information Technology (EIConCIT), Makassar, Indonesia, 2018, pp. 18-23.

[15] N. Veeraragavan, L. Arockiam and S. S. Manikandasaran, "Enhanced encryption algorithm (EEA) for protecting users' credentials in public cloud," 2017 International Conference on Algorithms, Methodology, Models and Applications in Emerging Technologies (ICAMMAET), Chennai, 2017, pp. 1-6.

[16] D. Pei, X. Guo and J. Zhang, "A video encryption service based on cloud computing," 2017 7th IEEE International Conference on Electronics Information and Emergency Communication (ICEIEC), Macau, 2017, pp. 167-171.

[17] S. Mudepalli, V. S. Rao and R. K. Kumar, "An efficient data retrieval approach using blowfish encryption on cloud ciphertext retrieval in cloud computing," 2017 International Conference on Intelligent Computing and Control Systems (ICICCS), Madurai, 2017, pp. 267-271.

[18] F. S. Wu, "Research of Cloud Platform Data Encryption Technology Based on ECC Algorithm," 2018 International Conference on Virtual Reality and Intelligent Systems (ICVRIS), Changsha, 2018, pp. 125-129.

[19] A. S. Awad, A. Yousif and G. Kadoda, "Enhanced Model for Cloud Data Security based on Searchable Encryption and Hybrid Fragmentation," 2019 International Conference on Computer, Control, Electrical, and Electronics Engineering (ICCCEEE), Khartoum, Sudan, 2019, pp. 1-4.

[20] Saha Rahul, Geetha G., Kumar Gulshan, Kim Tai-hoon "RK-AES: An Improved Version of AES Using a New Key Generation Process with Random Keys", Security and Communication Networks, Hindawi, 2018. 
[21] Digital Ocean. Available: https://www.digitalocean.com/ [Accessed: 11-May2020].

[22] Server Test, "Entropy and Randomness Online Tester." [Online]. Available:

https://servertest.online/entropy. [Acce

[23] F. Webster and S. E. Tavares, "On the Design of S-

Boxes," in Advances in Cryptology - CRYPTO '85

Proceedings, 1986, pp. 523-534. 